\title{
A new fractional Jacobi elliptic equation method for solving fractional partial differential equations
}

\section{Bin Zheng*}

\section{"Correspondence:}

zhengbin2601@126.com

School of Science, Shandong

University of Technology, Zibo,

Shandong 255049, China

\section{空 Springer}

\begin{abstract}
In this paper, we propose a new fractional Jacobi elliptic equation method to seek exact solutions of fractional partial differential equations. Based on a traveling wave transformation, certain fractional partial differential equation can be turned into another fractional ordinary differential equation. Then the fractional Jacobi elliptic equation is used as the auxiliary sub-equation to solve the fractional ordinary differential equation. As for applications of this method, we apply it to seek exact solutions for the space-time fractional Kortweg-de Vries (KdV) equation, the space-time fractional Benjamin-Bona-Mahony (BBM) equation, and the space-time fractional $(2+1)$-dimensional breaking soliton equations. With the aid of symbolic computation program, a series of exact solutions expressed in the Jacobi elliptic functions for the two equations are successfully found.

MSC: 35C07; 35Q53

Keywords: auxiliary sub-equation; fractional partial differential equation; exact solution; space-time fractional Kortweg-de Vries equation; space-time fractional Benjamin-Bona-Mahony equation; space-time fractional $(2+1)$-dimensional breaking soliton equations
\end{abstract}

\section{Introduction}

In recent decades, fractional differential equations have been paid an increasing attention as they are widely used to describe various complex phenomena in many fields such as the fluid flow, signal processing, control theory, systems identification, biology, and other areas. In particular, fractional derivative is useful in describing the memory and hereditary properties of materials and processes. Among the investigations for fractional differential equations, finding numerical and exact solutions to fractional differential equations is a hot topic. Many efficient methods have been proposed so far to obtain numerical solutions and exact solutions of fractional differential equations. For example, these methods include the fractional sub-equation method [1-7], the first integral method [8], the $\left(G^{\prime} / G\right)$ method [9-13], the variational iterative method [14-16], the Exp method [17], the simplest equation method [18], the Adomian decomposition method [19, 20], the homotopy perturbation method [21-23], the shifted Jacobi-Gauss-Lobatto collocation method [24], the shifted Legendre spectral method [25], the generalized Laguerre spectral algorithms [26], the modified generalized Laguerre tau method combining with a new operational matrix [27] and so on.

O2014 Zheng; licensee Springer. This is an Open Access article distributed under the terms of the Creative Commons Attribution License (http://creativecommons.org/licenses/by/2.0), which permits unrestricted use, distribution, and reproduction in any medium, provided the original work is properly cited. 
The Jacobi elliptic function method is an effective method for solving some fractional differential equations, which have been investigated in detail in $[28,29]$. In this paper, we propose a new fractional Jacobi elliptic equation method to seek exact solutions of fractional partial differential equations in the sense of the modified Riemann-Liouville derivative. First based on a traveling wave transformation, certain fractional partial differential equation can be turned into another fractional ordinary differential equation, the exact solutions of the latter are assumed to be expressed in a polynomial in $\left(\frac{D^{\alpha} G}{G}\right)$, where $D^{\alpha}$ denotes the modified Riemann-Liouville derivative of $\alpha$ order, and $G=G(\xi)$ satisfies the following fractional Jacobi elliptic equation:

$$
\left(D_{\xi}^{\alpha} G(\xi)\right)^{2}=e_{2} G^{4}(\xi)+e_{1} G^{2}(\xi)+e_{0}, \quad 0<\alpha \leq 1,
$$

where $e_{0}, e_{1}, e_{2}$ are arbitrary constants. The degree of the polynomial can be determined by the homogeneous balancing principle. By use of a fractional complex transformation, the general solutions of (1) can be obtained, with which the exact solutions for the original fractional partial differential equation can be deduced subsequently.

The definition and some important properties of the modified Riemann-Liouville derivative $[1-7,30]$ are listed as follows:

$$
\begin{aligned}
& D_{t}^{\alpha} f(t)= \begin{cases}\frac{1}{\Gamma(1-\alpha)} \frac{d}{d t} \int_{0}^{t}(t-\xi)^{-\alpha}(f(\xi)-f(0)) d \xi, & 0<\alpha<1, \\
\left(f^{(\alpha-n)}(t)\right)^{(n)}, & n \leq \alpha<n+1, n \geq 1,\end{cases} \\
& D_{t}^{\alpha} t^{r}=\frac{\Gamma(1+r)}{\Gamma(1+r-\alpha)} t^{r-\alpha}, \\
& D_{t}^{\alpha}(f(t) g(t))=g(t) D_{t}^{\alpha} f(t)+f(t) D_{t}^{\alpha} g(t), \\
& D_{t}^{\alpha} f[g(t)]=f_{g}^{\prime}[g(t)] D_{t}^{\alpha} g(t), \\
& D_{t}^{\alpha} f[g(t)]=D_{g}^{\alpha} f[g(t)]\left(g^{\prime}(t)\right)^{\alpha} .
\end{aligned}
$$

The rest of this paper is organized as follows. In Section 2, we give the description of the fractional Jacobi elliptic equation method for solving fractional partial differential equations. Then in Section 3 we apply this method to seek exact solutions for the space-time fractional KdV equation, the space-time fractional BBM equation, and the space-time fractional $(2+1)$-dimensional breaking soliton equations. Some concluding comments are presented at the end of this paper.

\section{Summary of the fractional Jacobi elliptic equation method}

In this section we give the description of the fractional Jacobi elliptic equation method for solving fractional partial differential equations.

Suppose that a fractional partial differential equation, say in the independent variables $t, x_{1}, x_{2}, \ldots, x_{n}$, is given by

$$
\begin{gathered}
P\left(u_{1}, \ldots u_{k}, D_{t}^{\alpha} u_{1}, \ldots, D_{t}^{\alpha} u_{k}, D_{x_{1}}^{\alpha} u_{1}, \ldots, D_{x_{1}}^{\alpha} u_{k}, \ldots, D_{x_{n}}^{\alpha} u_{1}, \ldots\right. \\
\left.D_{x_{n}}^{\alpha} u_{k}, D_{t}^{2 \alpha} u_{1}, \ldots, D_{t}^{2 \alpha} u_{k}, D_{x_{1}}^{2 \alpha} u_{1}, \ldots\right)=0
\end{gathered}
$$

where $u_{i}=u_{i}\left(t, x_{1}, x_{2}, \ldots, x_{n}\right), i=1, \ldots, k$ are unknown functions, $P$ is a polynomial in $u_{i}$ and their various partial derivatives including fractional derivatives. 
Step 1. Suppose

$$
u_{i}\left(t, x_{1}, x_{2}, \ldots, x_{n}\right)=U_{i}(\xi), \quad i=1, \ldots, k
$$

and a traveling wave transformation

$$
\xi=c t+k_{1} x_{1}+k_{2} x_{2}+\cdots+k_{n} x_{n}+\xi_{0} .
$$

Then by the property (6), (7) can be turned into the following fractional ordinary differential equation with respect to the variable $\xi$ :

$$
\begin{aligned}
& \widetilde{P}\left(U_{1}, \ldots, U_{k}, c^{\alpha} D_{\xi}^{\alpha} U_{1}, \ldots, c^{\alpha} D_{\xi}^{\alpha} U_{k}, k_{1}^{\alpha} D_{\xi}^{\alpha} U_{1}, \ldots, k_{1}^{\alpha} D_{\xi}^{\alpha} U_{k}, \ldots, k_{n}^{\alpha} D_{\xi}^{\alpha} U_{1}, \ldots,\right. \\
& \left.\quad k_{n}^{\alpha} D_{\xi}^{\alpha} U_{k}, c^{2 \alpha} D_{\xi}^{2 \alpha} U_{1}, \ldots, c^{2 \alpha} D_{\xi}^{2 \alpha} U_{k}, k_{1}^{2 \alpha} D_{\xi}^{2 \alpha} U_{1}, \ldots\right)=0 .
\end{aligned}
$$

Step 2. Suppose that the solution of (9) can be expressed by a polynomial in $\left(\frac{D_{\xi}^{\alpha} G}{G}\right)$ as follows:

$$
U_{j}(\xi)=\sum_{i=-m_{j}}^{m_{j}} a_{j, i}\left(\frac{D_{\xi}^{\alpha} G}{G}\right)^{i}, \quad j=1,2, \ldots, k
$$

where $a_{j, i}, i=-m_{j}, \ldots, 0,1, \ldots, m_{j}, j=1,2, \ldots, k$ are constants to be determined later, the positive integer $m_{j}$ can be determined by considering the homogeneous balance between the highest order derivatives and nonlinear terms appearing in (9), $G=G(\xi)$ satisfies the fractional Jacobi elliptic equation denoted by (1).

Step 3. Substituting (10) into (9) and using (1), collecting all terms with the same order of $\left(D_{\xi}^{\alpha} G\right)^{i} G^{j}$ together, the left-hand side of (9) is converted into another polynomial in $\left(D_{\xi}^{\alpha} G\right)^{i} G^{j}$. Equating each coefficient of this polynomial to zero, yields a set of algebraic equations for $a_{j, i}, i=-m_{j}, \ldots, 0,1, \ldots, m_{j}, j=1,2, \ldots, k$.

Step 4. Solving the equations system in Step 3, and using the general solutions of (1), we can construct a variety of exact solutions for (7).

In order to obtain the general expressions for $\left(\frac{D_{\xi}^{\alpha} G}{G}\right)$ in (1), we suppose $G(\xi)=H(\eta)$, and a nonlinear fractional complex transformation $\eta=\frac{\xi^{\alpha}}{\Gamma(1+\alpha)}$. Then by the properties (3) and (5), we have $D_{\xi}^{\alpha} G(\xi)=D_{\xi}^{\alpha} H(\eta)=H^{\prime}(\eta) D_{\xi}^{\alpha} \eta=H^{\prime}(\eta)$. So (1) can be turned into the following ordinary differential equation:

$$
\left(H^{\prime}(\eta)\right)^{2}=e_{2} H^{4}(\eta)+e_{1} H^{2}(\eta)+e_{0}
$$

By the general solutions of (11), one has

$$
\left(\frac{H^{\prime}(\eta)}{H(\eta)}\right)= \begin{cases}c n(\eta) d s(\eta), & e_{2}=m^{2}, e_{1}=-\left(1+m^{2}\right), e_{0}=0, \\ -s n(\eta) d c(\eta), & e_{2}=-m^{2}, e_{1}=2 m^{2}-1, e_{0}=1-m^{2}, \\ -m^{2} s n(\eta) c d(\eta), & e_{2}=-1, e_{1}=2-m^{2}, e_{0}=m^{2}-1, \\ -d c(\eta) n s(\eta), & e_{2}=1, e_{1}=2-m^{2}, e_{0}=1-m^{2}, \\ c s(\eta) n d(\eta), & e_{2}=m^{2}\left(m^{2}-1\right), e_{1}=2 m^{2}-1, e_{0}=1, \\ \left(1-m^{2}\right) s d(\eta) n c(\eta), & e_{2}=1, e_{1}=-\left(m^{2}+1\right), e_{0}=m^{2},\end{cases}
$$


where $\operatorname{sn}(\eta), c n(\eta), d n(\eta)$ denote the Jacobi elliptic sine function, Jacobi elliptic cosine function, and the Jacobi elliptic function of the third kind, respectively, $m$ is the modulus, and

$$
\begin{aligned}
& c s(\eta)=\frac{c n(\eta)}{s n(\eta)}, \quad s d(\eta)=\frac{s n(\eta)}{d n(\eta)}, \quad d c(\eta)=\frac{d n(\eta)}{c n(\eta)}, \\
& s c(\eta)=\frac{1}{c s(\eta)}, \quad d s(\eta)=\frac{1}{s d(\eta)}, \quad c d(\eta)=\frac{1}{d c(\eta)}, \\
& n d(\eta)=\frac{1}{d n(\eta)}, \quad n s(\eta)=\frac{1}{s n(\eta)}, \quad n c(\eta)=\frac{1}{c n(\eta)} .
\end{aligned}
$$

Furthermore, one can obtain the following expressions for $\frac{D_{\xi}^{\alpha} G(\xi)}{G(\xi)}$ :

$$
\begin{aligned}
& \left(\frac{D_{\xi}^{\alpha} G(\xi)}{G(\xi)}\right) \\
& \quad= \begin{cases}c n\left(\frac{\xi^{\alpha}}{\Gamma(1+\alpha)}\right) d s\left(\frac{\xi^{\alpha}}{\Gamma(1+\alpha)}\right), & e_{2}=m^{2}, e_{1}=-\left(1+m^{2}\right), e_{0}=0, \\
-\operatorname{sn}\left(\frac{\xi^{\alpha}}{\Gamma(1+\alpha)}\right) d c\left(\frac{\xi^{\alpha}}{\Gamma(1+\alpha)}\right), & e_{2}=-m^{2}, e_{1}=2 m^{2}-1, e_{0}=1-m^{2}, \\
-m^{2} \operatorname{sn}\left(\frac{\xi^{\alpha}}{\Gamma(1+\alpha)}\right) c d\left(\frac{\xi^{\alpha}}{\Gamma(1+\alpha)}\right), & e_{2}=-1, e_{1}=2-m^{2}, e_{0}=m^{2}-1, \\
-d c\left(\frac{\xi^{\alpha}}{\Gamma(1+\alpha)}\right) n s\left(\frac{\xi^{\alpha}}{\Gamma(1+\alpha)}\right), & e_{2}=1, e_{1}=2-m^{2}, e_{0}=1-m^{2}, \\
c s\left(\frac{\xi^{\alpha}}{\Gamma(1+\alpha)}\right) n d\left(\frac{\xi^{\alpha}}{\Gamma(1+\alpha)}\right), & e_{2}=m^{2}\left(m^{2}-1\right), e_{1}=2 m^{2}-1, e_{0}=1, \\
\left(1-m^{2}\right) \operatorname{sd}\left(\frac{\xi^{\alpha}}{\Gamma(1+\alpha)}\right) n c\left(\frac{\xi^{\alpha}}{\Gamma(1+\alpha)}\right), & e_{2}=1, e_{1}=-\left(m^{2}+1\right), e_{0}=m^{2} .\end{cases}
\end{aligned}
$$

Remark 1 For the sake of simplicity, other expressions for $\left(\frac{D_{\xi}^{\alpha} G(\xi)}{G(\xi)}\right)$ with $e_{2}, e_{1}, e_{0}$ taken different values are omitted here.

\section{Application of the fractional Jacobi elliptic equation method to some fractional partial differential equations}

\subsection{Space-time fractional KdV equation}

Consider the following space-time fractional KdV equation:

$$
D_{t}^{\alpha} u+30 u^{2} D_{x}^{\alpha} u+20\left(D_{x}^{\alpha} u\right)\left(D_{x}^{2 \alpha} u\right)+10 u D_{x}^{3 \alpha} u+D_{x}^{5 \alpha} u=0, \quad 0<\alpha \leq 1,
$$

which is a variation of the following $\mathrm{KdV}$ equation [31]:

$$
u_{t}+30 u^{2} u_{x}+20 u_{x} u_{x x}+10 u u_{x x x}+u_{x x x x x}=0 .
$$

In order to apply the fractional auxiliary sub-equation method described in Section 2, suppose $u(x, t)=U(\xi)$, where $\xi=c t+k x+\xi_{0}, k, c, \xi_{0}$ are all constants with $k, c \neq 0$. By use of (3) and (6), one has

$$
\left\{\begin{array}{l}
D_{t}^{\alpha} u=D_{t}^{\alpha} U(\xi)=D_{\xi}^{\alpha} U(\xi)\left(\xi_{t}^{\prime}(t)\right)^{\alpha}=c^{\alpha} D_{\xi}^{\alpha} U(\xi) \\
D_{x}^{\alpha} u=D_{x}^{\alpha} U(\xi)=D_{\xi}^{\alpha} U(\xi)\left(\xi_{x}^{\prime}(x)\right)^{\alpha}=k^{\alpha} D_{\xi}^{\alpha} U(\xi)
\end{array}\right.
$$

and then (14) can be turned into the following form:

$$
c^{\alpha} D_{\xi}^{\alpha} U+30 k^{\alpha} U^{2} D_{\xi}^{\alpha} U+20 k^{3 \alpha}\left(D_{\xi}^{\alpha} U\right)\left(D_{\xi}^{2 \alpha} U\right)+10 k^{3 \alpha} U D_{\xi}^{3 \alpha} U+k^{5 \alpha} D_{\xi}^{5 \alpha} U=0 .
$$


Suppose that the solution of (17) can be expressed by

$$
U(\xi)=\sum_{i=-m}^{m} a_{i}\left(\frac{D_{\xi}^{\alpha} G}{G}\right)^{i}
$$

where $G=G(\xi)$ satisfies (1). By Balancing the order between the highest order derivative term and nonlinear term in (17), we can obtain $m=2$. So we have

$$
U(\xi)=a_{0}+a_{1}\left(\frac{D_{\xi}^{\alpha} G}{G}\right)+a_{2}\left(\frac{D_{\xi}^{\alpha} G}{G}\right)^{2}+a_{-1}\left(\frac{D_{\xi}^{\alpha} G}{G}\right)^{-1}+a_{-2}\left(\frac{D_{\xi}^{\alpha} G}{G}\right)^{-2}
$$

Substituting (19) into (17), using (1), and collecting all the terms with the same power of $\left(D_{\xi}^{\alpha} G\right)^{i} G^{j}$ together, equating each coefficient to zero, yields a set of algebraic equations. Solving these equations with the aid of a symbolic computation program, yields the following results.

Case 1:

$$
\begin{aligned}
& a_{-2}=2 k^{2 \alpha}\left(-e_{1}^{2}+4 e_{0} e_{2}\right), \quad a_{-1}=0, \quad a_{1}=0, \quad a_{2}=0, \\
& a_{0}=\frac{40 k^{3 \alpha} e_{1} \pm \sqrt{-80 k^{6 \alpha} e_{1}^{2}-30 k^{\alpha} c^{\alpha}-960 k^{6 \alpha} e_{2} e_{0}}}{30 k^{\alpha}}
\end{aligned}
$$

Case 2:

$$
\begin{aligned}
& a_{-2}=0, \quad a_{-1}=0, \quad a_{1}=0, \quad a_{2}=-2 k^{2 \alpha}, \\
& a_{0}=\frac{40 k^{3 \alpha} e_{1} \pm \sqrt{-80 k^{6 \alpha} e_{1}^{2}-30 k^{\alpha} c^{\alpha}-960 k^{6 \alpha} e_{2} e_{0}}}{30 k^{\alpha}} .
\end{aligned}
$$

Substituting the results above into (19), and combining with (13) we can obtain a series of exact solutions in the forms of the Jacobi elliptic functions for (14). For example, from Case 1 we get the following exact solutions.

Family 1: when $e_{2}=m^{2}, e_{1}=-\left(1+m^{2}\right), e_{0}=0$,

$$
\begin{aligned}
u_{1}(x, t)= & \frac{-40 k^{3 \alpha}\left(1+m^{2}\right) \pm \sqrt{-80 k^{6 \alpha}\left(1+m^{2}\right)^{2}-30 k^{\alpha} c^{\alpha}}}{30 k^{\alpha}} \\
& -2 k^{2 \alpha}\left(1+m^{2}\right)^{2}\left[\operatorname{cn}\left(\frac{\xi^{\alpha}}{\Gamma(1+\alpha)}\right) d s\left(\frac{\xi^{\alpha}}{\Gamma(1+\alpha)}\right)\right]^{-2}
\end{aligned}
$$

where $\xi=c t+k x+\xi_{0}$.

Family 2: when $e_{2}=-m^{2}, e_{1}=2 m^{2}-1, e_{0}=1-m^{2}$,

$$
\begin{aligned}
u_{2}(x, t)= & \frac{40 k^{3 \alpha}\left(2 m^{2}-1\right) \pm \sqrt{-80 k^{6 \alpha}\left(2 m^{2}-1\right)^{2}-30 k^{\alpha} c^{\alpha}+960 k^{6 \alpha} m^{2}\left(1-m^{2}\right)}}{30 k^{\alpha}} \\
& -2 k^{2 \alpha}\left[\operatorname{sn}\left(\frac{\xi^{\alpha}}{\Gamma(1+\alpha)}\right) d c\left(\frac{\xi^{\alpha}}{\Gamma(1+\alpha)}\right)\right]^{-2},
\end{aligned}
$$

where $\xi=c t+k x+\xi_{0}$. 
Family 3: when $e_{2}=-1, e_{1}=2-m^{2}, e_{0}=m^{2}-1$,

$$
\begin{aligned}
u_{3}(x, t)= & \frac{40 k^{3 \alpha}\left(2-m^{2}\right) \pm \sqrt{-80 k^{6 \alpha}\left(2-m^{2}\right)^{2}-30 k^{\alpha} c^{\alpha}+960 k^{6 \alpha}\left(m^{2}-1\right)}}{30 k^{\alpha}} \\
& -2 k^{2 \alpha}\left[\operatorname{sn}\left(\frac{\xi^{\alpha}}{\Gamma(1+\alpha)}\right) c d\left(\frac{\xi^{\alpha}}{\Gamma(1+\alpha)}\right)\right]^{-2},
\end{aligned}
$$

where $\xi=c t+k x+\xi_{0}$.

Family 4: when $e_{2}=1, e_{1}=2-m^{2}, e_{0}=1-m^{2}$,

$$
\begin{aligned}
u_{4}(x, t)= & \frac{40 k^{3 \alpha}\left(2-m^{2}\right) \pm \sqrt{-80 k^{6 \alpha}\left(2-m^{2}\right)^{2}-30 k^{\alpha} c^{\alpha}+960 k^{6 \alpha}\left(m^{2}-1\right)}}{30 k^{\alpha}} \\
& -2 k^{2 \alpha} m^{4}\left[d c\left(\frac{\xi^{\alpha}}{\Gamma(1+\alpha)}\right) n s\left(\frac{\xi^{\alpha}}{\Gamma(1+\alpha)}\right)\right]^{-2},
\end{aligned}
$$

where $\xi=c t+k x+\xi_{0}$.

Family 5: when $e_{2}=m^{2}\left(m^{2}-1\right), e_{1}=2 m^{2}-1, e_{0}=1$,

$$
\begin{aligned}
u_{5}(x, t)= & \frac{40 k^{3 \alpha}\left(2 m^{2}-1\right) \pm \sqrt{-80 k^{6 \alpha}\left(2 m^{2}-1\right)^{2}-30 k^{\alpha} c^{\alpha}-960 k^{6 \alpha} m^{2}\left(m^{2}-1\right)}}{30 k^{\alpha}} \\
& -2 k^{2 \alpha}\left[\operatorname{cs}\left(\frac{\xi^{\alpha}}{\Gamma(1+\alpha)}\right) n d\left(\frac{\xi^{\alpha}}{\Gamma(1+\alpha)}\right)\right]^{-2},
\end{aligned}
$$

where $\xi=c t+k x+\xi_{0}$.

Family 6: when $e_{2}=1, e_{1}=-\left(m^{2}+1\right), e_{0}=m^{2}$,

$$
\begin{aligned}
u_{6}(x, t)= & \frac{-40 k^{3 \alpha}\left(m^{2}+1\right) \pm \sqrt{-80 k^{6 \alpha}\left(m^{2}+1\right)^{2}-30 k^{\alpha} c^{\alpha}-960 k^{6 \alpha} m^{2}}}{30 k^{\alpha}} \\
& -2 k^{2 \alpha}\left[s d\left(\frac{\xi^{\alpha}}{\Gamma(1+\alpha)}\right) n c\left(\frac{\xi^{\alpha}}{\Gamma(1+\alpha)}\right)\right]^{-2},
\end{aligned}
$$

where $\xi=c t+k x+\xi_{0}$.

From Case 2 we can also get some exact solutions expressed in the forms of the Jacobi elliptic functions for (14), which are omitted here.

\subsection{Space-time fractional BBM equation}

Consider the space-time fractional BBM equation

$$
D_{t}^{\alpha} u+u D_{x}^{\alpha} u+D_{x}^{\alpha} u-\mu D_{t}^{\alpha}\left(D_{x}^{2 \alpha} u\right)=0, \quad 0<\alpha \leq 1,
$$

which is a variation of the following BBM equation of integer order [32]:

$$
u_{t}+u u_{x}+u_{x}-\mu u_{x x t}=0
$$

Suppose $u(x, t)=U(\xi)$, where $\xi=c t+k x+\xi_{0}, k, c, \xi_{0}$ are all constants with $k, c \neq 0$. Then similar to the process of (16)-(17), (26) can be turned into the following form:

$$
c^{\alpha} D_{\xi}^{\alpha} U+k^{\alpha} U D_{\xi}^{\alpha} U+k^{\alpha} D_{\xi}^{\alpha} U-\mu c^{\alpha} k^{2 \alpha} D_{\xi}^{3 \alpha} U=0 .
$$


Suppose that the solution of (28) can be expressed by

$$
U(\xi)=\sum_{i=-m}^{m} a_{i}\left(\frac{D_{\xi}^{\alpha} G}{G}\right)^{i}
$$

where $G=G(\xi)$ satisfies (1). By Balancing the order between the highest order derivative term and nonlinear term in (28), we can obtain $m=2$. So we have

$$
U(\xi)=a_{0}+a_{1}\left(\frac{D_{\xi}^{\alpha} G}{G}\right)+a_{2}\left(\frac{D_{\xi}^{\alpha} G}{G}\right)^{2}+a_{-1}\left(\frac{D_{\xi}^{\alpha} G}{G}\right)^{-1}+a_{-2}\left(\frac{D_{\xi}^{\alpha} G}{G}\right)^{-2}
$$

Substituting (30) into (28), using (1), and collecting all the terms with the same power of $\left(D_{\xi}^{\alpha} G\right)^{i} G^{j}$ together, equating each coefficient to zero, yields a set of algebraic equations. Solving these equations yields the following two groups of values.

Case 1:

$$
\begin{aligned}
& a_{-2}=12 \mu c^{\alpha} k^{\alpha}\left(e_{1}^{2}-4 e_{2} e_{0}\right), \quad a_{-1}=0, \\
& a_{1}=0, \quad a_{2}=0, \quad a_{0}=-\frac{k^{\alpha}+8 \mu c^{\alpha} k^{2 \alpha} e_{1}+c^{\alpha}}{k^{\alpha}} .
\end{aligned}
$$

Case 2:

$$
a_{-2}=0, \quad a_{-1}=0, \quad a_{1}=0, \quad a_{2}=12 \mu c^{\alpha} k^{\alpha}, \quad a_{0}=-\frac{k^{\alpha}+8 \mu c^{\alpha} k^{2 \alpha} e_{1}+c^{\alpha}}{k^{\alpha}} .
$$

Substituting the results above into (30), and combining with (13) we can obtain a series of exact solutions in the forms of the Jacobi elliptic functions for (26).

From Case 1 we get the following exact solutions.

Family 1: when $e_{2}=m^{2}, e_{1}=-\left(1+m^{2}\right), e_{0}=0$,

$$
\begin{aligned}
u_{1}(x, t)= & -\frac{k^{\alpha}-8 \mu c^{\alpha} k^{2 \alpha}\left(1+m^{2}\right)+c^{\alpha}}{k^{\alpha}} \\
& +12 \mu c^{\alpha} k^{\alpha}\left(1+m^{2}\right)^{2}\left[c n\left(\frac{\xi^{\alpha}}{\Gamma(1+\alpha)}\right) d s\left(\frac{\xi^{\alpha}}{\Gamma(1+\alpha)}\right)\right]^{-2},
\end{aligned}
$$

where $\xi=c t+k x+\xi_{0}$.

Family 2: when $e_{2}=-m^{2}, e_{1}=2 m^{2}-1, e_{0}=1-m^{2}$,

$$
\begin{aligned}
u_{2}(x, t)= & -\frac{k^{\alpha}+8 \mu c^{\alpha} k^{2 \alpha}\left(2 m^{2}-1\right)+c^{\alpha}}{k^{\alpha}} \\
& +12 \mu c^{\alpha} k^{\alpha}\left[\operatorname{sn}\left(\frac{\xi^{\alpha}}{\Gamma(1+\alpha)}\right) d c\left(\frac{\xi^{\alpha}}{\Gamma(1+\alpha)}\right)\right]^{-2},
\end{aligned}
$$

where $\xi=c t+k x+\xi_{0}$.

Family 3 : when $e_{2}=-1, e_{1}=2-m^{2}, e_{0}=m^{2}-1$,

$$
\begin{aligned}
u_{3}(x, t)= & -\frac{k^{\alpha}+8 \mu c^{\alpha} k^{2 \alpha}\left(2-m^{2}\right)+c^{\alpha}}{k^{\alpha}} \\
& +12 \mu c^{\alpha} k^{\alpha}\left[\operatorname{sn}\left(\frac{\xi^{\alpha}}{\Gamma(1+\alpha)}\right) c d\left(\frac{\xi^{\alpha}}{\Gamma(1+\alpha)}\right)\right]^{-2},
\end{aligned}
$$

where $\xi=c t+k x+\xi_{0}$. 
Family 4: when $e_{2}=1, e_{1}=2-m^{2}, e_{0}=1-m^{2}$,

$$
\begin{aligned}
u_{4}(x, t)= & -\frac{k^{\alpha}+8 \mu c^{\alpha} k^{2 \alpha}\left(2-m^{2}\right)+c^{\alpha}}{k^{\alpha}} \\
& +12 \mu c^{\alpha} k^{\alpha} m^{4}\left[d c\left(\frac{\xi^{\alpha}}{\Gamma(1+\alpha)}\right) n s\left(\frac{\xi^{\alpha}}{\Gamma(1+\alpha)}\right)\right]^{-2},
\end{aligned}
$$

where $\xi=c t+k x+\xi_{0}$.

Family 5: when $e_{2}=m^{2}\left(m^{2}-1\right), e_{1}=2 m^{2}-1, e_{0}=1$,

$$
\begin{aligned}
u_{5}(x, t)= & -\frac{k^{\alpha}+8 \mu c^{\alpha} k^{2 \alpha}\left(2 m^{2}-1\right)+c^{\alpha}}{k^{\alpha}} \\
& +12 \mu c^{\alpha} k^{\alpha}\left[\operatorname{cs}\left(\frac{\xi^{\alpha}}{\Gamma(1+\alpha)}\right) n d\left(\frac{\xi^{\alpha}}{\Gamma(1+\alpha)}\right)\right]^{-2},
\end{aligned}
$$

where $\xi=c t+k x+\xi_{0}$.

Family 6: when $e_{2}=1, e_{1}=-\left(m^{2}+1\right), e_{0}=m^{2}$,

$$
\begin{aligned}
u_{6}(x, t)= & -\frac{k^{\alpha}-8 \mu c^{\alpha} k^{2 \alpha}\left(m^{2}+1\right)+c^{\alpha}}{k^{\alpha}} \\
& +12 \mu c^{\alpha} k^{\alpha}\left[s d\left(\frac{\xi^{\alpha}}{\Gamma(1+\alpha)}\right) n c\left(\frac{\xi^{\alpha}}{\Gamma(1+\alpha)}\right)\right]^{-2},
\end{aligned}
$$

where $\xi=c t+k x+\xi_{0}$.

From Case 2 we can also get some Jacobi elliptic function solutions for (26), which are omitted here.

Remark 2 The Jacobi elliptic function solutions (20)-(25) and (31)-(36) are new exact solutions for the space-time fractional $\mathrm{KdV}$ equation and the space-time fractional BBM equation respectively to the best of our knowledge.

\subsection{Space-time fractional $(2+1)$-dimensional breaking soliton equations}

Consider the following space-time fractional $(2+1)$-dimensional breaking soliton equations:

$$
\left\{\begin{array}{l}
\frac{\partial^{\alpha} u}{\partial t^{\alpha}}+a \frac{\partial^{3 \alpha} u}{\partial x^{2 \alpha} y^{\alpha}}+4 a u \frac{\partial^{\alpha} v}{\partial x^{\alpha}}+4 a \frac{\partial^{\alpha} u}{\partial x^{\alpha}} v=0, \quad 0<\alpha \leq 1, a \neq 0 \\
\frac{\partial^{\alpha} u}{\partial y^{\alpha}}=\frac{\partial^{\alpha} v}{\partial x^{\alpha}}
\end{array}\right.
$$

where the contained fractional derivative is the modified Riemann-Liouville derivative.

The corresponding integer order equation to (37) can be found in [33, 34]. Now we will apply the described method in Section 2 to solve (37). To begin with, we suppose $u(x, y, t)=U(\xi), v(x, y, t)=V(\xi)$, where $\xi=c t+k_{1} x+k_{2} y+\xi_{0}, k_{1}, k_{2}, c, \xi_{0}$ are all constants with $k_{1}, k_{2}, c \neq 0$. Then similar to the process of (16)-(17), (37) can be turned into the following form:

$$
\left\{\begin{array}{l}
c^{\alpha} D_{\xi}^{\alpha} U+a k_{1}^{2 \alpha} k_{2}^{\alpha} D_{\xi}^{3 \alpha} U+4 a k_{1}^{\alpha} U D_{\xi}^{\alpha} V+4 a k_{1}^{\alpha} V D_{\xi}^{\alpha} U=0, \\
k_{2}^{\alpha} D_{\xi}^{\alpha} U=k_{1}^{\alpha} D_{\xi}^{\alpha} V .
\end{array}\right.
$$


Suppose that the solution of (38) can be expressed by

$$
\left\{\begin{array}{l}
U(\xi)=\sum_{i=-m_{1}}^{m_{1}} a_{i}\left(\frac{G^{\prime}}{G}\right)^{i}, \\
V(\xi)=\sum_{i=-m_{2}}^{m_{2}} b_{i}\left(\frac{G^{\prime}}{G}\right)^{i}
\end{array}\right.
$$

where $G=G(\xi)$ satisfies (1). By balancing the order between the highest order derivative term and nonlinear term in (38), we can obtain $m_{1}=m_{2}=2$. So we have

$$
\left\{\begin{array}{l}
U(\xi)=a_{0}+a_{1}\left(\frac{D_{\xi}^{\alpha} G}{G}\right)+a_{2}\left(\frac{D_{\xi}^{\alpha} G}{G}\right)^{2}+a_{-1}\left(\frac{D_{\xi}^{\alpha} G}{G}\right)^{-1}+a_{-2}\left(\frac{D_{\xi}^{\alpha} G}{G}\right)^{-2}, \\
V(\xi)=b_{0}+b_{1}\left(\frac{G^{\prime}}{G}\right)+b_{2}\left(\frac{G^{\prime}}{G}\right)^{2}+b_{-1}\left(\frac{D_{\xi}^{\alpha} G}{G}\right)^{-1}+b_{-2}\left(\frac{D_{\xi}^{\alpha} G}{G}\right)^{-2} .
\end{array}\right.
$$

Substituting (40) into (38), using (1), and collecting all the terms with the same power of $\left(D_{\xi}^{\alpha} G\right)^{i} G^{j}$ together, equating each coefficient to zero, yields a set of algebraic equations. Solving these equations yields

$$
\begin{aligned}
& a_{0}=a_{0}, \quad a_{1}=0, \quad a_{2}=-\frac{3}{2} k_{1}^{2 \alpha}, \quad a_{-1}=0, \quad a_{-2}=0, \\
& b_{0}=-\frac{-8 a k_{1}^{2 \alpha} k_{2}^{\alpha} e_{1}+c^{\alpha}+4 a a_{0} k_{2}^{\alpha}}{4 a k_{1}^{\alpha}}, \quad b_{1}=0, \\
& b_{2}=-\frac{3}{2} k_{1}^{\alpha} k_{2}^{\alpha}, \quad b_{-1}=0, \quad b_{-2}=0,
\end{aligned}
$$

where $a_{0}$ is an arbitrary constant.

Substituting the results above into (40), and combining with (13) we can obtain a series of exact solutions in the forms of the Jacobi elliptic functions for (37).

Family 1: when $e_{2}=m^{2}, e_{1}=-\left(1+m^{2}\right), e_{0}=0$,

$$
\left\{\begin{array}{l}
u_{1}(x, y, t)=a_{0}-\frac{3}{2} k_{1}^{2 \alpha}\left[\operatorname{cn}\left(\frac{\xi^{\alpha}}{\Gamma(1+\alpha)}\right) d s\left(\frac{\xi^{\alpha}}{\Gamma(1+\alpha)}\right)\right]^{2}, \\
v_{1}(x, y, t)=-\frac{8 a k_{1}^{\alpha \alpha} k_{2}^{\alpha}\left(1+m^{2}\right)+c^{\alpha}+4 a a_{0} k_{2}^{\alpha}}{4 a k_{1}^{\alpha}}-\frac{3}{2} k_{1}^{\alpha} k_{2}^{\alpha}\left[\operatorname{cn}\left(\frac{\xi^{\alpha}}{\Gamma(1+\alpha)}\right) d s\left(\frac{\xi^{\alpha}}{\Gamma(1+\alpha)}\right)\right]^{2},
\end{array}\right.
$$

where $\xi=c t+k_{1} x+k_{2} y+\xi_{0}$.

Family 2: when $e_{2}=-m^{2}, e_{1}=2 m^{2}-1, e_{0}=1-m^{2}$,

$$
\left\{\begin{array}{l}
u_{2}(x, y, t)=a_{0}-\frac{3}{2} k_{1}^{2 \alpha}\left[\operatorname{sn}\left(\frac{\xi^{\alpha}}{\Gamma(1+\alpha)}\right) d c\left(\frac{\xi^{\alpha}}{\Gamma(1+\alpha)}\right)\right]^{2}, \\
v_{2}(x, y, t)=-\frac{-8 a k_{1}^{2 \alpha} k_{2}^{\alpha}\left(2 m^{2}-1\right)+c^{\alpha}+4 a a_{0} k_{2}^{\alpha}}{4 a k_{1}^{\alpha}}-\frac{3}{2} k_{1}^{\alpha} k_{2}^{\alpha}\left[\operatorname{sn}\left(\frac{\xi^{\alpha}}{\Gamma(1+\alpha)}\right) d c\left(\frac{\xi^{\alpha}}{\Gamma(1+\alpha)}\right)\right]^{2},
\end{array}\right.
$$

where $\xi=c t+k_{1} x+k_{2} y+\xi_{0}$.

Family 3: when $e_{2}=-1, e_{1}=2-m^{2}, e_{0}=m^{2}-1$,

$$
\left\{\begin{array}{l}
u_{3}(x, y, t)=a_{0}-\frac{3}{2} k_{1}^{2 \alpha} m^{4}\left[\operatorname{sn}\left(\frac{\xi^{\alpha}}{\Gamma(1+\alpha)}\right) c d\left(\frac{\xi^{\alpha}}{\Gamma(1+\alpha)}\right)\right]^{2}, \\
v_{3}(x, y, t)=-\frac{-8 a k_{1}^{2 \alpha} k_{2}^{\alpha}\left(2-m^{2}\right)+c^{\alpha}+4 a a_{0} k_{2}^{\alpha}}{4 a k_{1}^{\alpha}}-\frac{3}{2} k_{1}^{\alpha} k_{2}^{\alpha} m^{4}\left[\operatorname{sn}\left(\frac{\xi^{\alpha}}{\Gamma(1+\alpha)}\right) c d\left(\frac{\xi^{\alpha}}{\Gamma(1+\alpha)}\right)\right]^{2},
\end{array}\right.
$$

where $\xi=c t+k_{1} x+k_{2} y+\xi_{0}$.

Family 4: when $e_{2}=1, e_{1}=2-m^{2}, e_{0}=1-m^{2}$,

$$
\left\{\begin{array}{l}
u_{4}(x, y, t)=a_{0}-\frac{3}{2} k_{1}^{2 \alpha}\left[d c\left(\frac{\xi^{\alpha}}{\Gamma(1+\alpha)}\right) n s\left(\frac{\xi^{\alpha}}{\Gamma(1+\alpha)}\right)\right]^{2}, \\
v_{4}(x, y, t)=-\frac{-8 a k_{1}^{2 \alpha} k_{2}^{\alpha}\left(2-m^{2}\right)+c^{\alpha}+4 a a_{0} k_{2}^{\alpha}}{4 a k_{1}^{\alpha}}-\frac{3}{2} k_{1}^{\alpha} k_{2}^{\alpha}\left[d c\left(\frac{\xi^{\alpha}}{\Gamma(1+\alpha)}\right) n s\left(\frac{\xi^{\alpha}}{\Gamma(1+\alpha)}\right)\right]^{2},
\end{array}\right.
$$

where $\xi=c t+k_{1} x+k_{2} y+\xi_{0}$. 
Family 5: when $e_{2}=m^{2}\left(m^{2}-1\right), e_{1}=2 m^{2}-1, e_{0}=1$,

$$
\left\{\begin{array}{l}
u_{1}(x, y, t)=a_{0}-\frac{3}{2} k_{1}^{2 \alpha}\left[\operatorname{cs}\left(\frac{\xi^{\alpha}}{\Gamma(1+\alpha)}\right) n d\left(\frac{\xi^{\alpha}}{\Gamma(1+\alpha)}\right)\right]^{2}, \\
v_{1}(x, y, t)=-\frac{-8 a k_{1}^{2 \alpha} k_{2}^{\alpha}\left(2 m^{2}-1\right)+c^{\alpha}+4 a a_{0} k_{2}^{\alpha}}{4 a k_{1}^{\alpha}}-\frac{3}{2} k_{1}^{\alpha} k_{2}^{\alpha}\left[\operatorname{cs}\left(\frac{\xi^{\alpha}}{\Gamma(1+\alpha)}\right) n d\left(\frac{\xi^{\alpha}}{\Gamma(1+\alpha)}\right)\right]^{2}
\end{array}\right.
$$

where $\xi=c t+k_{1} x+k_{2} y+\xi_{0}$.

Family 6: when $e_{2}=1, e_{1}=-\left(m^{2}+1\right), e_{0}=m^{2}$,

$$
\left\{\begin{array}{l}
u_{1}(x, y, t)=a_{0}-\frac{3}{2} k_{1}^{2 \alpha}\left(1-m^{2}\right)^{2}\left[s d\left(\frac{\xi^{\alpha}}{\Gamma(1+\alpha)}\right) n c\left(\frac{\xi^{\alpha}}{\Gamma(1+\alpha)}\right)\right]^{2}, \\
v_{1}(x, y, t)=-\frac{8 a k_{1}^{2 \alpha} k_{2}^{\alpha}\left(m^{2}+1\right)+c^{\alpha}+4 a a_{0} k_{2}^{\alpha}}{4 a k_{1}^{\alpha}}-\frac{3}{2} k_{1}^{\alpha} k_{2}^{\alpha}\left(1-m^{2}\right)^{2}\left[s d\left(\frac{\xi^{\alpha}}{\Gamma(1+\alpha)}\right) n c\left(\frac{\xi^{\alpha}}{\Gamma(1+\alpha)}\right)\right]^{2},
\end{array}\right.
$$

where $\xi=c t+k_{1} x+k_{2} y+\xi_{0}$.

Remark 3 We note that the Jacobi elliptic function solutions established in (41)-(46) for the space-time fractional $(2+1)$-dimensional breaking soliton equations are new exact solutions so far in the literature.

Remark 4 Combining with other general solutions of the Jacobi elliptic equation (1) where $e_{2}, e_{1}, e_{0}$ taken different values, one can obtain abundant different exact solutions from those listed above for the space-time fractional $\mathrm{KdV}$ equation, the space-time fractional BBM equation, and the space-time fractional $(2+1)$-dimensional breaking soliton equations, which are omitted here for the sake of simplicity.

\section{Conclusions}

In this paper, we have proposed a new approach for seek exact solutions of fractional partial differential equations in the sense of the modified Riemann-Liouville derivative. Based the fractional Jacobi elliptic equation, exact traveling wave solutions in the forms of the Jacobi elliptic functions can be obtained for fractional partial differential equations. For illustrating the validity of this method, we apply it to seek exact solutions for two fractional equations: the space-time fractional $\mathrm{KdV}$ equation, the space-time fractional BBM equation, and the space-time fractional $(2+1)$-dimensional breaking soliton equations. As a result, a series of explicit solutions expressed in the Jacobi elliptic functions for them are successfully found with the aid of symbolic computation program. Being concise and powerful, we note that this method can also be applied to solve many other fractional partial differential equations arising in mathematical physics.

Competing interests

The author declares that they have no competing interests.

Author's contributions

BZ carried out the research of this article and read and approved the final manuscript.

\section{Acknowledgements}

The author thanks the referees very much for their careful comments and valuable suggestions on this paper. This work was partially supported by the Natural Science Foundation of Shandong Province (in China) (Grant no. ZR2013AQ009). 


\section{References}

1. Zhang, S, Zhang, HQ: Fractional sub-equation method and its applications to nonlinear fractional PDEs. Phys. Lett. A 375, 1069-1073 (2011)

2. Guo, SM, Mei, LQ, Li, Y, Sun, YF: The improved fractional sub-equation method and its applications to the space-time fractional differential equations in fluid mechanics. Phys. Lett. A 376, 407-411 (2012)

3. Lu, B: Bäcklund transformation of fractional Riccati equation and its applications to nonlinear fractional partial differential equations. Phys. Lett. A 376, 2045-2048 (2012)

4. Tang, B, He, YN, Wei, LL, Zhang, XD: A generalized fractional sub-equation method for fractional differential equations with variable coefficients. Phys. Lett. A 376, 2588-2590 (2012)

5. Wen, $C B$, Zheng, B: A new fractional sub-equation method for fractional partial differential equations. WSEAS Trans. Math. 12(5), 564-571 (2013)

6. Meng, FW: A new approach for solving fractional partial differential equations. J. Appl. Math. 2013, Article ID 256823 (2013)

7. Zheng, B, Wen, CB: Exact solutions for fractional partial differential equations by a new fractional sub-equation method. Adv. Differ. Equ. 2013, 199 (2013)

8. Lu, B: The first integral method for some time fractional differential equations. J. Math. Anal. Appl. 395, 684-693 (2012)

9. Gepreel, KA, Omran, S: Exact solutions for nonlinear partial fractional differential equations. Chin. Phys. B 21(11), $110204(2012)$

10. Akgül, A, Kılıçman, A, Inc, M: Improved ( $\left.G^{\prime} / G\right)$-expansion method for the space and time fractional foam drainage and KdV equations. Abstr. Appl. Anal. 2013, Article ID 414353 (2013)

11. Zheng, B: Exact solutions for some fractional partial differential equations by the $\left(G^{\prime} / G\right)$ method. Math. Probl. Eng. 2013, Article ID 826369 (2013)

12. Zheng, B: ( $\left.G^{\prime} / G\right)$-Expansion method for solving fractional partial differential equations in the theory of mathematical physics. Commun. Theor. Phys. 58, 623-630 (2012)

13. Bekir, A, Güner, Ö: Exact solutions of nonlinear fractional differential equations by $\left(G^{\prime} / G\right)$-expansion method. Chin. Phys. B 22(11), 110202 (2013)

14. He, JH: A new approach to nonlinear partial differential equations. Commun. Nonlinear Sci. Numer. Simul. 2, 230-235 (1997)

15. Wu, GC: A fractional variational iteration method for solving fractional nonlinear differential equations. Comput. Math. Appl. 61(8), 2186-2190 (2011)

16. Guo, S, Mei, L: The fractional variational iteration method using He's polynomials. Phys. Lett. A 375, 309-313 (2011)

17. Zheng, B: Exp-function method for solving fractional partial differential equations. Sci. World J. 2013, Article ID $465723(2013)$

18. Taghizadeh, N, Mirzazadeh, M, Rahimian, M, Akbari, M: Application of the simplest equation method to some time-fractional partial differential equations. Ain Shams Eng. J. 4, 897-902 (2013)

19. El-Sayed, AMA, Gaber, M: The Adomian decomposition method for solving partial differential equations of fractal order in finite domains. Phys. Lett. A 359, 175-182 (2006)

20. El-Sayed, AMA, Behiry, SH, Raslan, WE: Adomian's decomposition method for solving an intermediate fractional advection-dispersion equation. Comput. Math. Appl. 59, 1759-1765 (2010)

21. Guo, S, Mei, L, Li, Y: Fractional variational homotopy perturbation iteration method and its application to a fractional diffusion equation. Appl. Math. Comput. 219, 5909-5917 (2013)

22. He, JH: Homotopy perturbation technique. Comput. Methods Appl. Mech. Eng. 178, 257-262 (1999)

23. $\mathrm{He}, \mathrm{JH}$ : A coupling method of homotopy technique and a perturbation technique for non-linear problems. Int. J. Non-Linear Mech. 35, 37-43 (2000)

24. Bhrawy, AH, Alghamdi, MA: A shifted Jacobi-Gauss-Lobatto collocation method for solving nonlinear fractional Langevin equation involving two fractional orders in different intervals. Bound. Value Probl. 2012, 62 (2012)

25. Bhrawy, AH, Al-Shomrani, MM: A shifted Legendre spectral method for fractional-order multi-point boundary value problems. Adv. Differ. Equ. 2012, 8 (2012)

26. Baleanu, D, Bhrawy, AH, Taha, TM: Two efficient generalized Laguerre spectral algorithms for fractional initial value problems. Abstr. Appl. Anal. 2013, Article ID 546502 (2013)

27. Bhrawy, AH, Alghamdi, MM, Taha, TM: A new modified generalized Laguerre operational matrix of fractional integration for solving fractional differential equations on the half line. Adv. Differ. Equ. 2012, 179 (2012)

28. Bhrawy, AH, Tharwat, MM, Yildirim, A, Abdelkawy, MA: A Jacobi elliptic function method for nonlinear arrays of vortices. Indian J. Phys. 86(12), 1107-1113 (2012)

29. Bhrawy, AH, Abdelkawy, MA, Biswas, A: Cnoidal and snoidal wave solutions to coupled nonlinear wave equations by the extended Jacobi's elliptic function method. Commun. Nonlinear Sci. Numer. Simul. 18, 915-925 (2013)

30. Jumarie, G: Table of some basic fractional calculus formulae derived from a modified Riemann-Liouville derivative for non-differentiable functions. Appl. Math. Lett. 22, 378-385 (2009)

31. Salas, $\mathrm{AH}$ : Exact solutions for the general fifth $\mathrm{KdV}$ equation by the exp function method. Appl. Math. Comput. 205 291-297 (2008)

32. Benjamin, TB, Bona, JL, Mahony, J: Model equations for long waves in nonlinear dispersive systems. Philos. Trans. R. Soc. Lond. 272, 47-48 (1972)

33. Zhang, S: New exact non-traveling wave and coefficient function solutions of the $(2+1)$-dimensional breaking soliton equations. Phys. Lett. A 368, 470-475 (2007)

34. Peng, YZ, Krishnan, EV: Two classes of new exact solutions to $(2+1)$-dimensional breaking soliton equation Commun. Theor. Phys. 44, 807-809 (2005)

10.1186/1687-1847-2014-228

Cite this article as: Zheng: A new fractional Jacobi elliptic equation method for solving fractional partial differential equations. Advances in Difference Equations 2014, 2014:228 